\title{
Editorial
}

\section{Questioning orthodoxy}

$\mathrm{R}$ ecently hearing Fred Singer from the USA lecture on what he perceives to be the uncritical ways in which global change has been attributed to anthropogenic effects reminded me of the importance we should attach to those who question our current beliefs. For Fred it was not sufficient that the IPCC had engaged many of the best scientific brains in the world to reach the existing consensus; they might all be wrong because the original question or assumption was wrong. Fred was strongly challenged by the audience of Antarctic scientists, not least because some of his quotations were selective in order to initiate discussion. And we know that there are areas of considerable weakness amongst the several proxies used to compute the rate of temperature change, that we have only poorly quantified and modelled the role of clouds, energy transfer between the oceans and atmosphere, water vapour as a greenhouse gas and that we have yet to be certain that the Global Climate Models really do have all the most significant driving variables. So the IPCC conclusions are drawn on the best available evidence with complementary patterns derived from several different approaches and constitute the best we can do at the moment.

Fred's comments and questions were all aimed at the details of the science and its interpretation and we can debate them as scientists. Not so those of some other recent questioners. Bjorn Lomberg's recent attempt to set global change in a priority list of global problems using a panel of economists - the Copenhagen Consensus - is clearly an attack of a different nature. Here we are faced with an unrealistic attempt to decide on an either/or basis between questions set out one dimensionally. Who cares if there were Nobel laureates amongst the group if the question they were addressing was the wrong one? This is not questioning orthodoxy but simply a blatant attempt to push global change down the priority pile by making inappropriate comparisons with suspect methodology. The panel admitted that cost/benefit analysis was difficult to apply to the wide range of questions they addressed so how can they conclude that money is better spent on AIDS prevention (which might reduce the expected death toll over the next 6-8 years below 30 million) when one billion people are suffering and dying now from inadequate water supplies? What is the value of providing clean water if you do not also have adequate sanitation and health care? If drought supervenes because of global change how then will the water be provided or food grown? Biasing the approach towards easily identifiable and costed projects gives them a spurious current value when compared to multi-faceted longterm projects that cannot be as easily costed. And global change was represented in this list as the Kyoto Protocol and carbon tax. The final listing from the Copenhagen Consensus ignores the inter-relatededness of the topics, their relative importance to different groups and the fact that we have to address all of them at the same time if we want to make civilisation work better.

In listening to those who question our science and the conclusions we try to draw from it we need to understand the motives of the questioners. Of course we may be wrong in detail and we should always be clear about the quality of the data and the robustness of the conclusions. We must however be prepared to take a stand when others try to misrepresent the problem and diminish the efforts the science community has made to sketch out a likely scenario for the future of the world. The Copenhagen Consensus of eight seems a rather small group when compared to the IPCC cast of thousands.

DAVID WALTON 